\title{
${ }^{13}$ C NMR Relaxation of iso-Poly(2-vinylpyridinium chloride) in Aqueous Solution. Effects of Electrostatic Interactions on Local Chain Dynamics
}

\author{
Shinichi Yamazaki, ${ }^{\dagger}$ Eisaku Okada, Yoshio Muroga, \\ Ichiro NoDA, and Akihiro TsUTSUMI* \\ Department of Applied Chemistry, Graduate School of Engineering, Nagoya University, \\ Furo-cho, Chikusa-ku, Nagoya 464-8603, Japan \\ * Department of Applied Physics, Faculty of Engineering, Hokkaido University, \\ Kita-13, Nishi-8, Kita-ku, Sapporo 060-8628, Japan
}

(Received March 16, 1999)

\begin{abstract}
Measurements of ${ }^{13} \mathrm{C}$ spin-lattice relaxation times, $T_{1}$, and nuclear Overhauser effects, NOE, were carried out as a function of temperature at two magnetic fields for iso-poly(2-vinylpyridine) (iso-P2VP) in 1,4-dioxane- $d_{8}$ and iso-poly(2-vinylpyridinium chloride) (iso- $\mathrm{P} 2 \mathrm{VPCl}$ ), in $\mathrm{D}_{2} \mathrm{O}$ to investigate the effects of electrostatic interactions on local chain dynamics. For main chain carbons of both polymers, the experimental $T_{1}$ and NOE data are in good agreement with the Dejean-Lauprêtre-Monnerie (DLM) model in the entire temperature range. It was found that the local conformational transitions of main chain and the libration are more restricted for iso-P2VPCl than iso-P2VP. For pyridyl ring carbons, the experimental data were reproduced by the models combining the DLM model and restricted ring rotations. The angular amplitude $\alpha$ of ring rotation of iso-P2 VPCl is smaller than that of iso-P2VP. These results imply that both local motions of main chain and side chain are affected by the electrostatic interactions.

KEY WORDS ${ }^{13} \mathrm{C}$ Nuclear Magnetic Resonance Relaxation / Poly(2-vinylpyridine) / Poly(2vinylpyridinium chloride) / Electrostatic Interactions / Local Chain Dynamics / Spin-Lattice Relaxation Time / Nuclear Overhauser Effect /
\end{abstract}

Effects of electrostatic interactions on local chain conformation or chain stiffness have been extensively studied theoretically and experimentally. ${ }^{1-4}$ However, few studies have been reported on their effects on local chain dynamics.

Among various dynamic methods such as NMR relaxation, ${ }^{5}$ dielectric relaxation, ${ }^{6,7}$ and time-resolved fluorescence methods, ${ }^{8}$ the NMR relaxation method is a very powerful technique because it provides detailed information about molecular motions of different parts of chain through the spectral density function, which is the Fourier transformation of correlation function of the relaxing dipoles in different environments of chain.

Poly(2-vinylpyridine) (P2VP) and poly(4-vinylpyridine) (P4VP) are suitable model polyelectrolytes because they can be converted to ionized polymer from nonionic one by neutralizing pyridyl ring by relevant acid without the significant change of chemical structure. Chachaty et al. studied the local chain dynamics of P2VP and P4VP, and their ionized polymers, poly(2-vinylpyridinium chloride) ( $\mathrm{P} 2 \mathrm{VPCl}$ ) and poly(4-vinylpyridinium bromide) $(\mathrm{P} 4 \mathrm{VPBr})$ in solutions and reported that electrostatic interactions affect local chain dynamics. ${ }^{9-11}$ However, the effects of electrostatic interactions remain unclear yet, since the chain motions were not analyzed by reliable motional models such as conformational jump models in their papers.

Recently, Ravindranathan et al. studied the local chain dynamics of ata-P2VP in chloroform, ${ }^{12}$ and reported that all the experimental data of main chain carbons are in good agreement with the Dejean-Lauprêtre-Monnerie (DLM) model, ${ }^{13}$ which was modified from the Hall-

\footnotetext{
$\dagger$ To whom correspondence should be addressed.
}

Weber-Helfand (HWH) model describing the main chain motions in terms of the conformational transitions $\mathrm{s}^{14,15}$ by introducing libration motion of $\mathrm{C}-\mathrm{H}$ vector. In a previous paper, we also reported that the DLM model is well applied to the main chain motions of poly $(t$-butyl crotonate) in toluene, of which a $\beta$-methyl group makes the chain stiff. ${ }^{16}$ For pyridyl ring motion of ata-P2VP, on the other hand, Ravindranathan et al. reported that the experimental data are well reproduced by the model combining the HWH model of main chain and the strongly restricted rotation of pyridyl ring. In this work, therefore, we measured ${ }^{13} \mathrm{C}$ NMR relaxation such as spin-lattice relaxation time $T_{1}$ and nuclear Overhauser effect, NOE, of iso-P2VP and iso- $\mathrm{P} 2 \mathrm{VPCl}$ at two magnetic fields as a function of temperature and analyzed these data of main chains and pyridyl rings by the DLM model and the restricted rotational model to study the effects of electrostatic interactions on the local chain dynamics. Here, we used iso-P2VP and iso- $\mathrm{P} 2 \mathrm{VPCl}$ as the samples because the tacticity may affect the local chain dynamics.

\section{EXPERIMENTAL}

iso-P2VP used here was prepared by anionic polymerization initiated by phenylmagnesium bromide in our laboratory. Details of the preparation were described elsewhere. ${ }^{17}$ The tacticities of iso-P2VP thus obtained are almost isotactic $(\mathrm{I}+\mathrm{H}=96.5 \%, \mathrm{~S}=3.5 \%$, where $\mathrm{I}, \mathrm{H}$, and $\mathrm{S}$ represent isotactic, heterotactic, and syndiotactic, respectively) determined by methine peak intensity of ${ }^{1} \mathrm{H}$ NMR spectra. ${ }^{18}$ The molecular weight is $c a$. 82000 determined by viscosity measurement assuming that the viscosity equation of ata-P2 $\mathrm{VP}^{19}$ is 
${ }^{13} \mathrm{C}$ NMR Relaxation of iso-P2VPCl

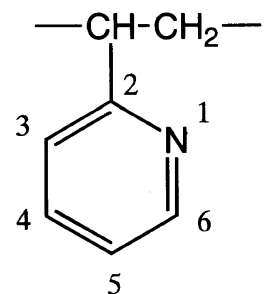

Figure 1. Numbering of the atoms in a pyridyl ring of the repeating unit of P2VP.

applicable to iso-P2VP. This assumption can be satisfactorily accepted because the viscosity equation of ata-P2VP is obtained in a typical good solvent, 1,4dioxane. Moreover, the effects of molecular weight on relaxation parameters such as $T_{1}$ and NOE are almost negligible if the molecular weight exceeds $c a .10000 .{ }^{9}$ iso- $\mathrm{P} 2 \mathrm{VPCl}$ solution was prepared by neutralizing pyridyl ring by $\mathrm{HCl}$ in $\mathrm{D}_{2} \mathrm{O}$ purchased from Aldrich Chemical Co. On the other hand, iso-P2VP was dissolved in 1,4-dioxane- $d_{8}$ purchased from Aldrich Chemical Co. The polymer concentrations were fixed at $10 \mathrm{wt} / \mathrm{vol} \%$ in both solutions. Both solutions were degassed carefully and sealed in sample tubes. Atoms in a pyridyl ring are numbered as shown in Figure 1.

${ }^{13} \mathrm{C}$ NMR relaxation experiments were performed with Varian unity INOVA 500, Varian Mercury and JEOL FX-270 NMR instruments operating at 125.7, 75.5, and $67.8 \mathrm{MHz}$ for carbon resonance frequency, respectively. Temperatures were regulated within $\pm 1 \mathrm{~K}$ by a controller. $T_{1}$ data were measured by the conventional inversion recovery technique using $180^{\circ}-\tau-90^{\circ}$ pulse sequence. A total of about 500 acquisitions was accumulated for a set of 12-14 arrayed $\tau$ values and the delay times between pulse cycles were 5 times longer than maximum $T_{1}$. The $T_{1}$ value was determined by fitting the observed data to a nonlinear exponential function. NOE data were evaluated as the ratios of ${ }^{13} \mathrm{C}$ signal intensities obtained by complete ${ }^{1} \mathrm{H}$ decoupling to those obtained by inverse gated decoupling. The delay times for NOE experiments were 10 times longer than maximum $T_{1}$. The relative errors of measured $T_{1}$ and NOE values were estimated to be lower than 10 and $15 \%$, respectively.

\section{THEORETICAL BACKGROUND}

Assuming a purely ${ }^{13} \mathrm{C}-{ }^{1} \mathrm{H}$ dipolar-dipolar relaxation mechanism, we have the following equations for the spin-lattice relaxation time, $T_{1}$ dd, and nuclear Overhauser effect, NOE, under the complete proton decoupling. ${ }^{20}$

$$
\begin{aligned}
\frac{1}{T_{1}^{\mathrm{dd}}}= & \frac{N}{10}\left[\frac{\mu_{0} \hbar \gamma_{\mathrm{H}} \gamma_{\mathrm{C}}}{4 \pi r^{3}}\right]^{2}\left[J\left(\omega_{\mathrm{H}}-\omega_{\mathrm{C}}\right)\right. \\
& \left.+3 J\left(\omega_{\mathrm{C}}\right)+6 J\left(\omega_{\mathrm{H}}+\omega_{\mathrm{C}}\right)\right] \\
\mathrm{NOE}=1+ & \frac{\gamma_{\mathrm{H}}}{\gamma_{\mathrm{C}}}\left[\frac{6 J\left(\omega_{\mathrm{H}}+\omega_{\mathrm{C}}\right)-J\left(\omega_{\mathrm{H}}-\omega_{\mathrm{C}}\right)}{J\left(\omega_{\mathrm{H}}-\omega_{\mathrm{C}}\right)+3 J\left(\omega_{\mathrm{C}}\right)+6 J\left(\omega_{\mathrm{H}}+\omega_{\mathrm{C}}\right)}\right]
\end{aligned}
$$

where $N, \mu_{0}$, and $r$ are the number of direct bonded proton, the permeability in vacuum, the $\mathrm{C}-\mathrm{H}$ internuclear distance $\left(0.109 \mathrm{~nm}\right.$ for aliphatic carbons ${ }^{21}$ and $0.108 \mathrm{~nm}$ for pyridyl ring carbons, ${ }^{22}$ respectively), respectively, $\hbar=h / 2 \pi$ with $h$ being Planck constant, and $\gamma_{\mathrm{H}}, \gamma_{\mathrm{C}}, \omega_{\mathrm{H}}$, and $\omega_{\mathrm{C}}$ are the magnetogyric ratios and Larmor frequencies of ${ }^{1} \mathrm{H}$ and ${ }^{13} \mathrm{C}$, respectively.

For the carbons of pyridyl ring, the chemical shift anisotropy should be taken into account in addition to the dipole-dipole relaxation as below ${ }^{23}$

$$
\begin{gathered}
\frac{1}{T_{1}}=\frac{1}{T_{1}^{\mathrm{dd}}}+\frac{1}{T_{1}^{\mathrm{CSA}}} \\
\mathrm{NOE}=1+\frac{\gamma_{\mathrm{H}}}{\gamma_{\mathrm{C}}}\left[\frac{N}{10}\left(\frac{\mu_{0} \hbar \gamma_{\mathrm{H}} \gamma_{\mathrm{C}}}{4 \pi r^{3}}\right)^{2}\right. \\
\left.\times\left\{\frac{6 J\left(\omega_{\mathrm{H}}+\omega_{\mathrm{C}}\right)-J\left(\omega_{\mathrm{H}}-\omega_{\mathrm{C}}\right)}{\left(T_{1}^{\mathrm{dd}}\right)^{-1}+\left(T_{1}^{\mathrm{CSA}}\right)^{-1}}\right\}\right]
\end{gathered}
$$

where $T_{1}^{\mathrm{CSA}}$ is the relaxation time arisen from chemical shift anisotropy and given by, if the chemical shift is axially symmetric, ${ }^{23}$

$$
\frac{1}{T_{1}^{\mathrm{CSA}}}=\frac{2}{15} \omega_{\mathrm{C}}^{2}\left(\sigma_{\|}-\sigma_{\perp}\right)^{2} J\left(\omega_{\mathrm{C}}\right)
$$

where $\sigma_{\|}$and $\sigma_{\perp}$ are the components of the shift tensor, parallel and perpendicular to the symmetric axis. The magnitude of $\left(\sigma_{\|}-\sigma_{\perp}\right)$ is obtained from the principal values of ${ }^{13} \mathrm{C}$ chemical shift tensor as

$$
\sigma_{\|}-\sigma_{\perp}=\sigma_{33}-\frac{1}{2}\left(\sigma_{11}+\sigma_{22}\right)
$$

In calculations, the value for both polymers is assumed to be equal to $197 \mathrm{ppm}$ obtained for the $\mathrm{C}_{4}$ carbon of pyridine. ${ }^{24}$ This assumption is considered not to make serious errors in the calculation at least at the low magnetic field because the contribution of chemical shift anisotropy is much smaller than that of dipole-dipole relaxation.

The spectral density function, $J(\omega)$, is obtained by the Fourier transformation of the correlation function, $G(t)$, which is derived on the basis of motional models.

$$
J(\omega)=\frac{1}{2} \int_{-\infty}^{\infty} G(t) \exp (i \omega t) d t
$$

\section{MOTIONAL MODELS}

\section{Main Chain Motion}

Although various models for the local motion of main chain of a polymer have been proposed, one of the most useful model may be the Dejean-Lauprêtre-Monnerie (DLM) model ${ }^{13}$ describing local chain motions in terms of conformational transition and libration, which well explains $T_{1}$ and NOE data of various polymers in solution and bulk. The spectral density function, $J(\omega)$, of the DLM model is given by

$$
J(\omega)=\frac{1-f}{(\alpha+i \beta)^{1 / 2}}+\frac{f \tau_{2}}{1+\omega^{2} \tau_{2}^{2}}
$$

with

$$
\alpha=\frac{1}{\tau_{0}^{2}}+\frac{2}{\tau_{0} \tau_{1}}-\omega^{2}
$$


and

$$
\beta=-2 \omega\left[\frac{1}{\tau_{0}}+\frac{1}{\tau_{1}}\right]
$$

where $\tau_{0}, \tau_{1}, \tau_{2}$, and $f$ are the correlation times for damping, propagation and libration, and the relative weight of libration, respectively. According to the theory of Howarth, ${ }^{25}$ moreover, $f$ is given by

$$
1-f=\left[\frac{\cos \theta-\cos ^{3} \theta}{2(1-\cos \theta)}\right]^{2}
$$

where $\theta$ is the libration angle of $\mathrm{C}-\mathrm{H}$ vector.

\section{Ring Rotational Motion}

The relaxation parameters, $T_{1}$ and NOE, in pyridyl ring carbons are given by superposing both segmental motions in main chain and rotational motions in the pyridyl ring. According to Ghesquière et al., ${ }^{10,11}$ the rotational motions of pyridyl rings of $\mathrm{P} 4 \mathrm{VP}$ and $\mathrm{P} 4 \mathrm{VPBr}$ are well described by the restricted rotational model such as the oscillation with small amplitude. Since the ring rotations of iso- $\mathrm{P} 2 \mathrm{VP}$ and iso- $\mathrm{P} 2 \mathrm{VPCl}$ are considered to be similar to those of $\mathrm{P} 4 \mathrm{VP}$ and $\mathrm{P} 4 \mathrm{VPBr}$, our experimental data were also analyzed by the same model. The superposed spectral density function is given by ${ }^{10}$

$$
\begin{aligned}
J(\omega)= & \frac{3}{8}\left\{\left[\frac{2}{3}\left(1-3 \cos ^{2} \gamma\right)^{2}+\sin ^{2} 2 \gamma(1+\cos \alpha)\right.\right. \\
& \left.+\sin ^{4} \gamma(1+\cos 2 \alpha)\right] J\left(\tau_{0}, \omega\right)+\left[\sin ^{2} 2 \gamma(1-\cos \alpha)\right. \\
& \left.\left.+\sin ^{4} \gamma(1-\cos 2 \alpha)\right] J\left(\tau_{t}, \omega\right)\right\}
\end{aligned}
$$

where $\gamma$ is the angle between the internuclear vector and the axis of rotation and $\alpha$ is the angular amplitude of oscillation. Here, we assumed that the temperature dependence of the amplitude of oscillation is given by $\alpha=C T^{1 / 2}$, which was employed for the phenyl ring motions by Gronski et al. ${ }^{26}$ If the main chain motions are described by the DLM model, $J\left(\tau_{0}, \omega\right)$ is given by eq 8 and $J\left(\tau_{t}, \omega\right)$ is given by replacing $\tau_{0}$ in eq 8 by $\tau_{t}$ as

$$
\tau_{t}^{-1}=\tau_{0}^{-1}+\tau_{\mathrm{ir}}^{-1}
$$

where $\tau_{\text {ir }}$ denotes the internal correlation time for pyridyl ring rotation.

\section{FITTING PROCEDURE}

According to Helfand ${ }^{27}$ who applied the theory of $\mathrm{Kramer}^{28}$ for the diffusion of a particle over a potential barrier to conformational transitions of polymer chains, the temperature dependence of the correlation time $\tau(T)$ is given by the Arrhenius type as

$$
\tau(T)=A \exp \left(E_{\mathrm{a}} / R T\right)
$$

with $A=\eta c$ where $\eta, c$, and $E_{\mathrm{a}}$ are the solvent viscosity, a molecular constant and the apparent activation energy, respectively. In the fitting procedure, $A$ and $E_{\mathrm{a}}$ were treated as adjustable parameters.

The $T_{1}$ and NOE data are fitted to the motional models simultaneously at the two magnetic fields as a function of temperature. At first the parameters, $A, E_{\mathrm{a}}, f, \tau_{0} / \tau_{1}$, 616 (a) iso- $\mathrm{P} 2 \mathrm{VPCl}$

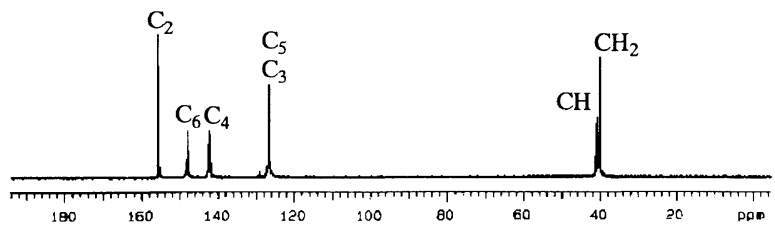

(b) iso-P2VP

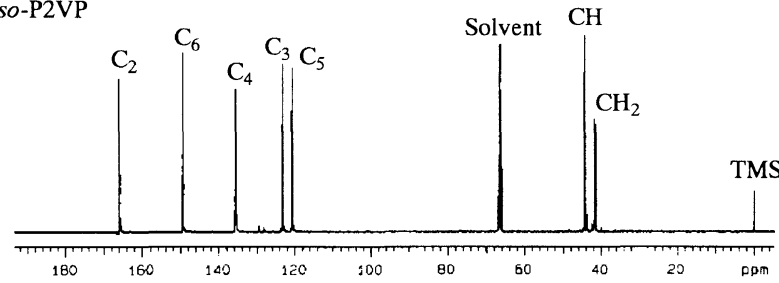

Figure 2. ${ }^{13} \mathrm{C}$ NMR spectra of (a) iso- $\mathrm{P} 2 \mathrm{VPCl}$ and (b) iso-P2VP at $125.7 \mathrm{MHz}$.

and $\tau_{1} / \tau_{2}$ in the DLM model were fitted manually for main chain carbons, and then the parameters of the ring rotation model were fitted for the pyridyl ring carbons while the fitting parameters obtained for the main chain carbons were fixed.

\section{RESULTS}

Figure 2 shows the ${ }^{13} \mathrm{C}$ NMR spectra of iso-P2VPCl (a) and iso-P2VP (b) at $125.7 \mathrm{MHz}$. The chemical shift was measured from a relevant reference and the peaks were assigned in Figure 2 as reported previously. ${ }^{9}$ Since the $\mathrm{C}_{3}$ and $\mathrm{C}_{5}$ carbons peaks of iso- $\mathrm{P} 2 \mathrm{VPCl}$ are overlapped each other, the $T_{1}$ and NOE values of these carbons could not be accurately determined. In this work, therefore, we examined the segmental motions of main chain carbons and the rotational motions of pyridyl ring $\left(\mathrm{C}_{4}\right.$ and $\mathrm{C}_{6}$ carbons).

Tables I and II summarize the ${ }^{13} \mathrm{C} T_{1}$ and NOE data as a function of temperature at the two magnetic fields for iso- $\mathrm{P} 2 \mathrm{VPCl}$ and iso-P2VP, respectively. These data indicate the following specific features. For the main chain carbons of both polymers, the larger magnetic fields are, the larger the $T_{1}$ values are, and the $T_{1}$ data pass through $T_{1}$ minimums and increase with increasing temperature at both magnetic fields, especially at the high magnetic field. Moreover, the NOE values are significantly smaller than the maximum value (3.0) at the extreme narrowing limit and decrease with increasing magnetic fields. Apparently, these results indicate that a single exponential correlation function, i.e., an isotropic motional model, cannot be applied to the data. The ratios of $T_{1}$ values for methine and methylene carbons, $T_{1}(\mathrm{CH}) / T_{1}\left(\mathrm{CH}_{2}\right)$, are $2.0 \pm 0.1$ and $1.8 \pm 0.1$ for iso$\mathrm{P} 2 \mathrm{VPCl}$ and iso-P2VP, respectively. Since the ratio for iso- $\mathrm{P} 2 \mathrm{VPCl}$ is equal to 2 , which is equal to the ratio of the number of directly bonded protons, the local motions for methine and methylene carbons are almost identical for iso- $\mathrm{P} 2 \mathrm{VPCl}$, while the local motions of $\mathrm{C}-\mathrm{H}$ vector are significantly different between the methine and methylene carbons of iso-P2VP.

The $T_{1}$ values of the pyridyl ring carbons $\left(\mathrm{C}_{4}\right.$ and $\mathrm{C}_{6}$ carbons) for iso- $\mathrm{P} 2 \mathrm{VPCl}$ and iso-P2VP are larger than 
Table I. Experimental $T_{1}(\mathrm{~ms})$ and $\mathrm{NOE}^{\mathrm{a}}$ data of iso- $\mathrm{P} 2 \mathrm{VPCl}$ in $\mathrm{D}_{2} \mathrm{O}$ as a function of temperature at two magnetic fields

\begin{tabular}{|c|c|c|c|c|c|c|c|c|}
\hline \multirow{3}{*}{ Temp/K } & \multicolumn{4}{|c|}{ Main chain carbons } & \multicolumn{4}{|c|}{ Pyridyl ring carbons } \\
\hline & \multicolumn{2}{|c|}{$\mathrm{CH}$} & \multicolumn{2}{|c|}{$\mathrm{CH}_{2}$} & \multicolumn{2}{|c|}{$\mathrm{C}_{4}$} & \multicolumn{2}{|c|}{$\mathrm{C}_{6}$} \\
\hline & $125.7 \mathrm{MHz}$ & $75.5 \mathrm{MHz}$ & $125.7 \mathrm{MHz}$ & $75.5 \mathrm{MHz}$ & $125.7 \mathrm{MHz}$ & $75.5 \mathrm{MHz}$ & $125.7 \mathrm{MHz}$ & $75.5 \mathrm{MHz}$ \\
\hline 303 & $290(1.61)$ & $187(1.83)$ & $147(1.60)$ & $99(1.82)$ & $229(1.59)$ & $179(1.78)$ & $201(1.51)$ & $162(1.71)$ \\
\hline 313 & $296(1.85)$ & $177(1.89)$ & $152(1.92)$ & $101(2.00)$ & $234(1.80)$ & $186(1.76)$ & $192(1.71)$ & $173(1.80)$ \\
\hline 333 & $335(2.13)$ & $208(2.25)$ & $161(2.10)$ & $115(2.17)$ & $261(1.83)$ & $213(2.00)$ & $222(1.77)$ & $206(1.88)$ \\
\hline 343 & $361(2.01)$ & $239(2.35)$ & $173(2.12)$ & $126(2.28)$ & $274(2.00)$ & $238(2.11)$ & $245(1.93)$ & $232(1.92)$ \\
\hline 353 & $367(2.15)$ & $229(2.31)$ & $194(2.17)$ & $135(2.34)$ & $284(1.99)$ & $260(2.13)$ & $259(2.00)$ & $238(2.05)$ \\
\hline
\end{tabular}

${ }^{\text {a }}$ Values in parentheses.

Table II. Experimental $T_{1}(\mathrm{~ms})$ and $\mathrm{NOE}^{\mathrm{a}}$ data of iso-P2VP in 1,4-dioxane- $d_{8}$ as a function of temperature at two magnetic fields

\begin{tabular}{|c|c|c|c|c|c|c|c|c|}
\hline \multirow[b]{2}{*}{ Temp/K } & \multicolumn{4}{|c|}{ Main chain carbons } & \multicolumn{4}{|c|}{ Pyridyl ring carbons } \\
\hline & \multicolumn{2}{|c|}{$\mathrm{CH}$} & \multicolumn{2}{|c|}{$\mathrm{CH}_{2}$} & \multicolumn{2}{|c|}{$\mathrm{C}_{4}$} & \multicolumn{2}{|c|}{$\mathrm{C}_{6}$} \\
\hline 303 & $330(1.44)$ & $167(1.72)$ & $177(1.46)$ & $92(1.70)$ & $266(1.33)$ & $185(1.68)$ & $289(1.48)$ & $200(1.68)$ \\
\hline 313 & $310(1.51)$ & $179(1.81)$ & $172(1.51)$ & $92(1.83)$ & $257(1.39)$ & $201(1.74)$ & $277(1.47)$ & $216(1.78)$ \\
\hline 323 & $302(1.58)$ & $181(1.87)$ & $164(1.59)$ & $97(1.90)$ & $256(1.44)$ & $210(1.73)$ & $277(1.58)$ & $220(1.88)$ \\
\hline 333 & $307(1.64)$ & $186(2.02)$ & $167(1.60)$ & $105(2.08)$ & $261(1.51)$ & $230(1.96)$ & $284(1.64)$ & $244(1.98)$ \\
\hline 343 & $312(1.74)$ & $210(1.98)$ & $172(1.74)$ & $111(2.23)$ & $273(1.57)$ & $248(2.00)$ & $291(1.70)$ & $255(1.99)$ \\
\hline 353 & $324(1.82)$ & $230(2.08)$ & $173(1.84)$ & $121(2.12)$ & $290(1.65)$ & $279(2.26)$ & $313(1.87)$ & $280(2.02)$ \\
\hline
\end{tabular}

${ }^{a}$ Values in parentheses.

those of main chain carbons at the low magnetic field, while the former values are smaller than the latter values at the high magnetic field. These facts imply that the motions of pyridyl ring contribute the $T_{1}$ values in addition to the segmental motions in the main chain and the chemical shift anisotropy plays an important role for the relaxation of pyridyl ring carbons, especially at the high magnetic field. Since the $T_{1}$ values for $\mathrm{C}_{4}$ and $\mathrm{C}_{6}$ carbons of both polymers are almost the same, the motion of pyridyl ring can be represented by the rotational motion around $\mathrm{C}_{2}-\mathrm{C}_{5}$ axis.

\section{DISCUSSION}

Figures 3, 4, 5, and 6 show the best fitting curves of $T_{1}$ and NOE vs. temperature, calculated by the parameters of the DLM model listed in Table III for methine and methylene carbons of iso- $\mathrm{P} 2 \mathrm{VPCl}$ and iso-P2VP, respectively. In the entire temperature range, the experimental $T_{1}$ and NOE values are in good agreement with the DLM model for both polymers. As shown in Table III, the correlation time ratio $\tau_{0} / \tau_{1}$ of iso- $\mathrm{P} 2 \mathrm{VPCl}$ (5) is smaller than that of iso-P2VP (8). The smaller correlation time ratio means that the propagation of local motion associated with $\tau_{1}$ is more restricted by damping associated with $\tau_{0}$ for iso- $\mathrm{P} 2 \mathrm{VPCl}$ than for iso-P2VP.

Moreover, let us examine the activation energy $E^{*}$ associated with a potential barrier for conformational transition. If eq 14 is valid for the conformational transitions, $E^{*}$ is given by
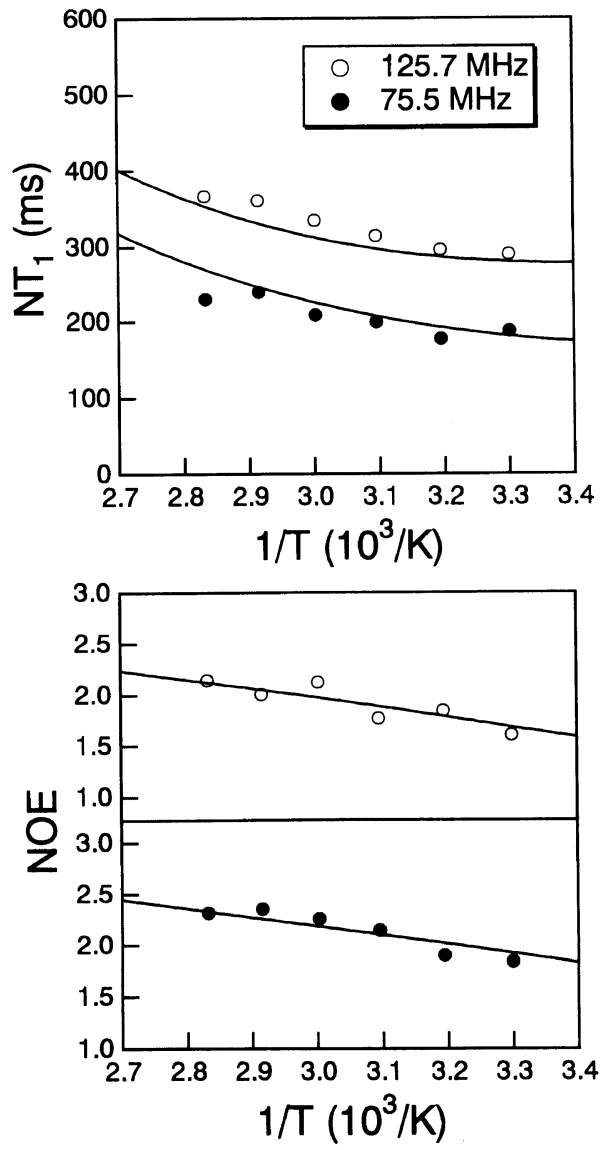

Figure 3. Temperature dependences of $T_{1}$ and NOE for methine carbon of iso-P2VPCl. Solid lines are calculated by the DLM model with the parameters listed in Table III. 

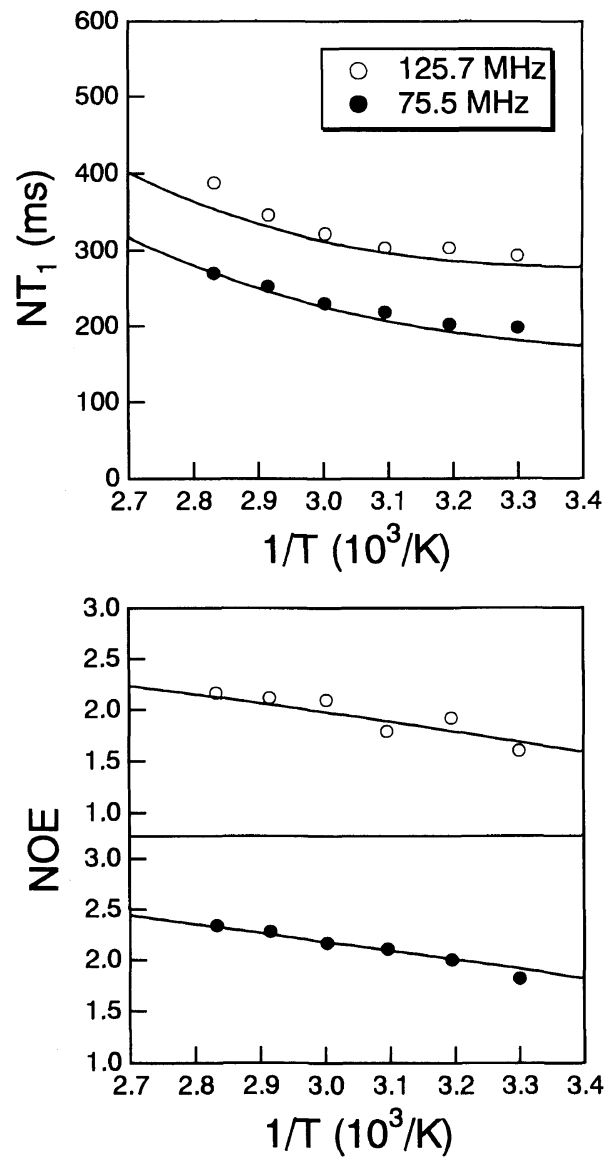

Figure 4. Temperature dependences of $T_{1}$ and NOE for methylene carbon of iso-P2 VPCl. Solid lines are calculated by the DLM model with the parameters listed in Table III

$$
E^{*}=E_{\mathrm{a}}-E_{\eta}
$$

where $E_{\eta}$ is the activation energy of solvent viscosity, which is expressed as $\eta=B \exp \left(E_{\eta} / R T\right)$ with $B$ being a constant. As reported by Gisser et al. ${ }^{29}$ however, eq 14 is not valid for ordinary flexible chains, and their correlation times are proportional to $\eta^{k}(k=0.41)$. In this case we have

$$
E^{*}=E_{\mathrm{a}}-k E_{\eta}
$$

Using $E_{\eta}=15.6$ and $12.5 \mathrm{~kJ} \mathrm{~mol}^{-1}$ for water and 1,4-dioxane, respectively, ${ }^{30}$ we have $E^{*}=2.6$ and $6.8 \mathrm{~kJ} \mathrm{~mol}^{-1}$ by eq 15 , and $E^{*}=11.8$ and $14.2 \mathrm{~kJ} \mathrm{~mol}^{-1}$ by eq 16 for iso-P2VPCl and iso-P2VP, respectively. In either case the activation energy of conformational transition of main chain is smaller for iso-P2 $\mathrm{VPCl}$ than for iso-P2VP. Combining the results of $E^{*}$ and $\tau_{0} / \tau_{1}$, we may conclude that the main chain motions of iso- $\mathrm{P} 2 \mathrm{VPCl}$ are more restricted than those of iso-P2VP by the electrostatic interactions between charges on pyridyl rings since $\tau_{0} / \tau_{1}$ can be assumed to be independent of solvent viscosity, while both the propagation and damping rates are larger for iso- $\mathrm{P} 2 \mathrm{VPCl}$ than for iso-P2VP.

The libration angles, $\theta$, are the same for methine and methylene carbons of iso- $\mathrm{P} 2 \mathrm{VPCl}$. This result is consistent with the fact that the value of $T_{1}(\mathrm{CH}) / T_{1}\left(\mathrm{CH}_{2}\right)$ is almost equal to 2. For iso-P2VP, on the other hand, the $\theta$ values are significantly different between methine and methylene carbons, so that the value of $T_{1}(\mathrm{CH})$ /
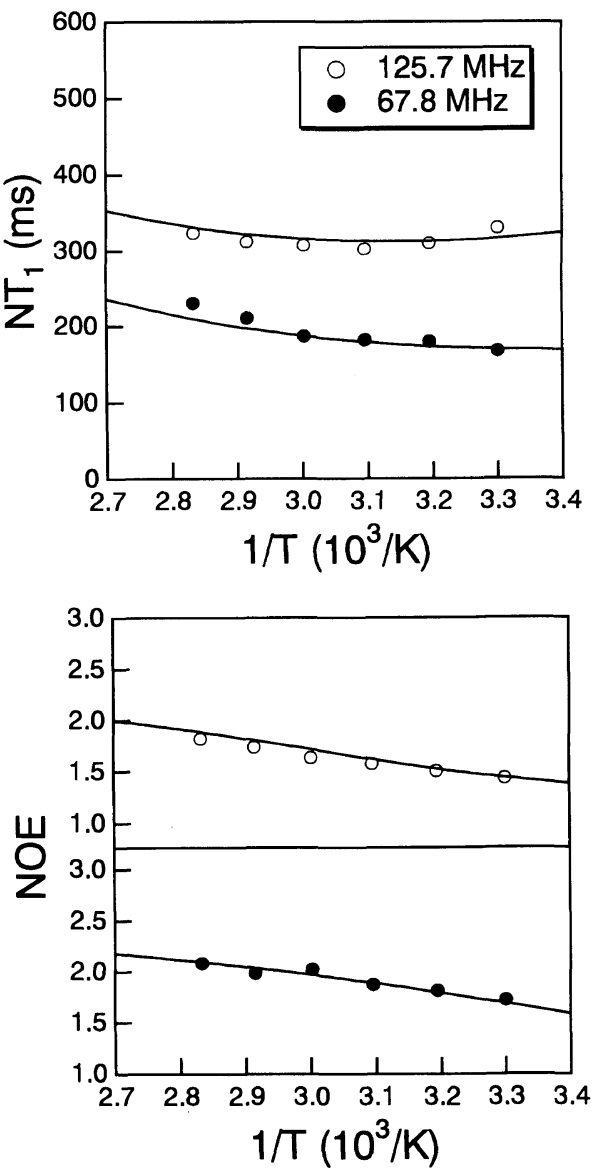

Figure 5. Temperature dependences of $T_{1}$ and NOE for methine carbon of iso-P2VP. Solid lines are calculated by the DLM model with the parameters listed in Table III.

$T_{1}\left(\mathrm{CH}_{2}\right)$ is different from $2(1.8 \pm 0.1)$. Moreover, the $\theta$ value of iso- $\mathrm{P} 2 \mathrm{VPCl}$ is always smaller than that of iso-P2VP. Thus, we may conclude that the libration is also restricted by the electrostatic interactions, since the libration motion is so fast that it is not significantly affected by solvent viscosity. ${ }^{29,31}$

According to the method of Guillermo et al., ${ }^{32}$ $\log \left(N T_{1} / \omega_{c}\right)$ is plotted against $\log \left(\omega_{c} \tau_{1}\right)$ for methine carbons of iso-P2VPCl and iso-P2VP at the two Larmor frequencies in Figure 7. The $T_{1}$ data superpose fairly well for both polymers. The superposition implies that the mechanisms of local motions as reflected in the correlation function are independent of temperature, and it justifies the above analysis.

Figures 8 and 9 show the temperature dependence of $T_{1}$ and NOE data for pyridyl ring carbon $\left(\mathrm{C}_{4}\right)$ of iso- $\mathrm{P} 2 \mathrm{VPCl}$ and iso-P2VP, respectively. Solid lines in these figures denote the theoretical values calculated by the restricted rotational model (eq 12) using the parameters listed in Table IV. As shown in these figures, the $T_{1}$ and NOE data of both polymers are almost satisfactorily reproduced by the model. The model parameters thus obtained are remarkably different between iso-P2VPCl and iso-P2VP; the angular amplitude of oscillation $\alpha$ for iso- $\mathrm{P} 2 \mathrm{VPCl}\left(28-30^{\circ}\right)$ is smaller than that for iso-P2VP $\left(37-39^{\circ}\right)$, though their pre-factor $A_{\text {ir }}$ and activation energy $E_{\text {ir }}$ are not much different. This result implies that the ring rotation of iso- $\mathrm{P} 2 \mathrm{VPCl}$ is more restricted than that of iso-P2VP by electrostatic 

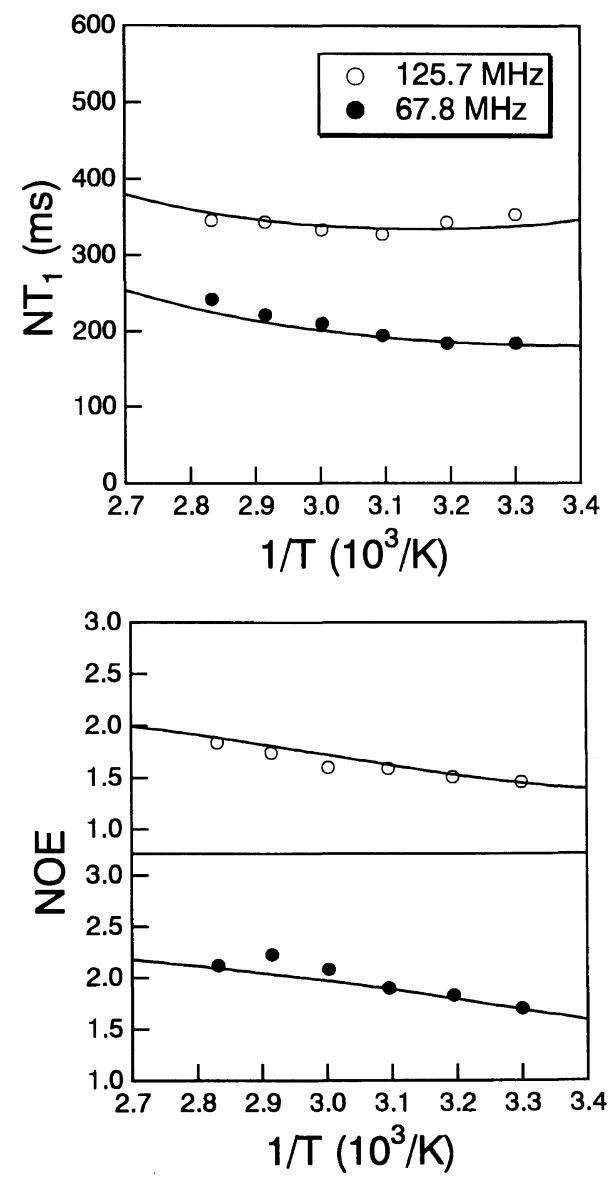

Figure 6. Temperature dependences of $T_{1}$ and NOE for methylene carbon of iso-P2VP. Solid lines are calculated by the DLM model with the parameters listed in Table III.

Table III. Fitting parameters of the DLM model for main chain carbons of iso- $\mathrm{P} 2 \mathrm{VPCl}$ and iso- $\mathrm{P} 2 \mathrm{VP}$

\begin{tabular}{llccccc}
\hline & & $\tau_{0} / \tau_{1}$ & $\tau_{1} / \tau_{2}$ & $E_{\mathrm{a}} / \mathrm{kJ} \mathrm{mol}^{-1}$ & $10^{12} A / \mathrm{s}$ & $\theta / \mathrm{deg}$ \\
\hline P2VPCl & Methine & 5 & 100 & 18.2 & 0.50 & 20.0 \\
& Methylene & 5 & 100 & 18.2 & 0.50 & 20.0 \\
P2VP & Methine & 8 & 100 & 19.3 & 0.60 & 24.0 \\
& Methylene & 8 & 100 & 19.3 & 0.60 & 27.0 \\
\hline
\end{tabular}

interactions if the solvent effects are not significant for the amplitude.

Finally, we will briefly discuss the effect of tacticities on the local chain dynamics. Comparing our fitting parameters with those of Ravindranathan et al., ${ }^{12}$ we found the significant difference in the libration angles: our data are $24.0^{\circ}$ and $27.0^{\circ}$ for methine and methylene carbons, respectively, while their data are $21.3^{\circ}$ and $25.7^{\circ}$. The larger libration angles of iso-P2VP imply that the effects of pyridyl ring on the libration motion are smaller for iso-P2VP than for ata-P2VP, because the pyridyl rings are oriented in the same directions for the former.

Acknowledgment. We are indebted to Prof. Toshifumi Hiraoki for helping us in NMR measurements at Hokkaido University.

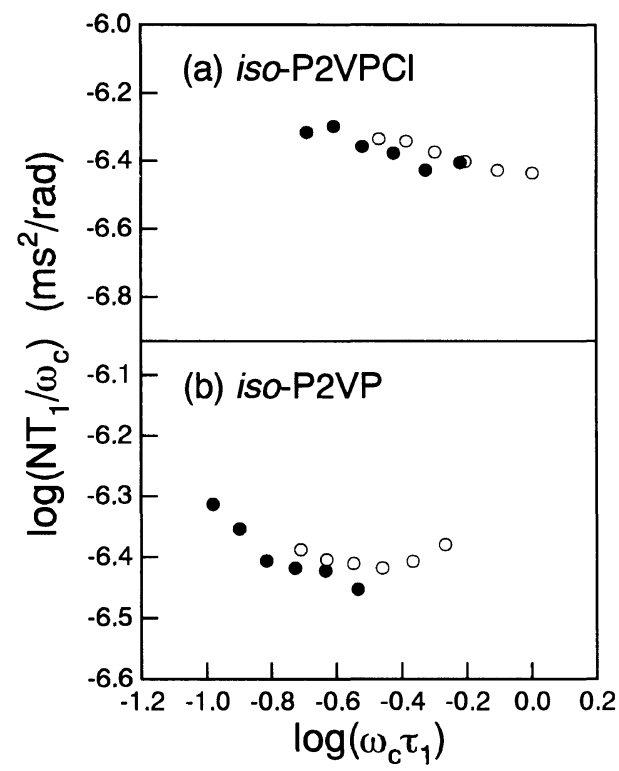

Figure 7. Frequency-temperature superposition of ${ }^{13} \mathrm{C} N T_{1}$ values for the methine carbons of iso- $\mathrm{P} 2 \mathrm{VPCl}$ (a) and iso-P2VP (b). Open and filled symbols denote the data of higher and lower magnetic fields, respectively. The $\tau_{1}$ values were evaluated by using the DLM model.
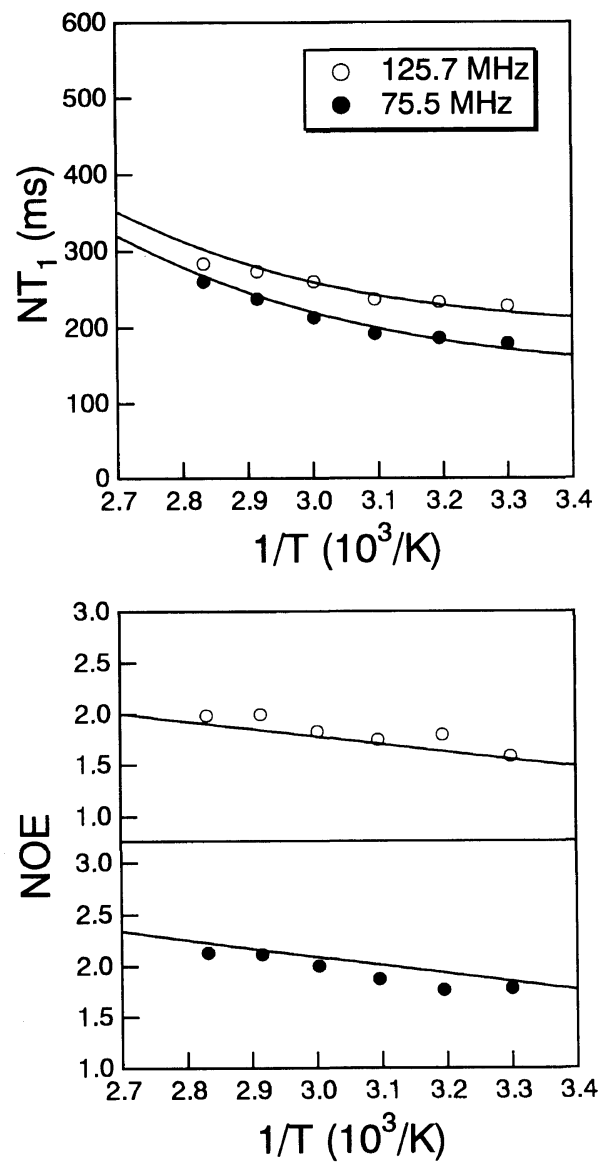

Figure 8. Temperature dependences of $T_{1}$ and NOE for $\mathrm{C}_{4}$ carbon in pyridyl ring of iso-P2VPCl. Solid lines denote the theoretical values of the restricted rotational model with the parameters listed in Table IV. 

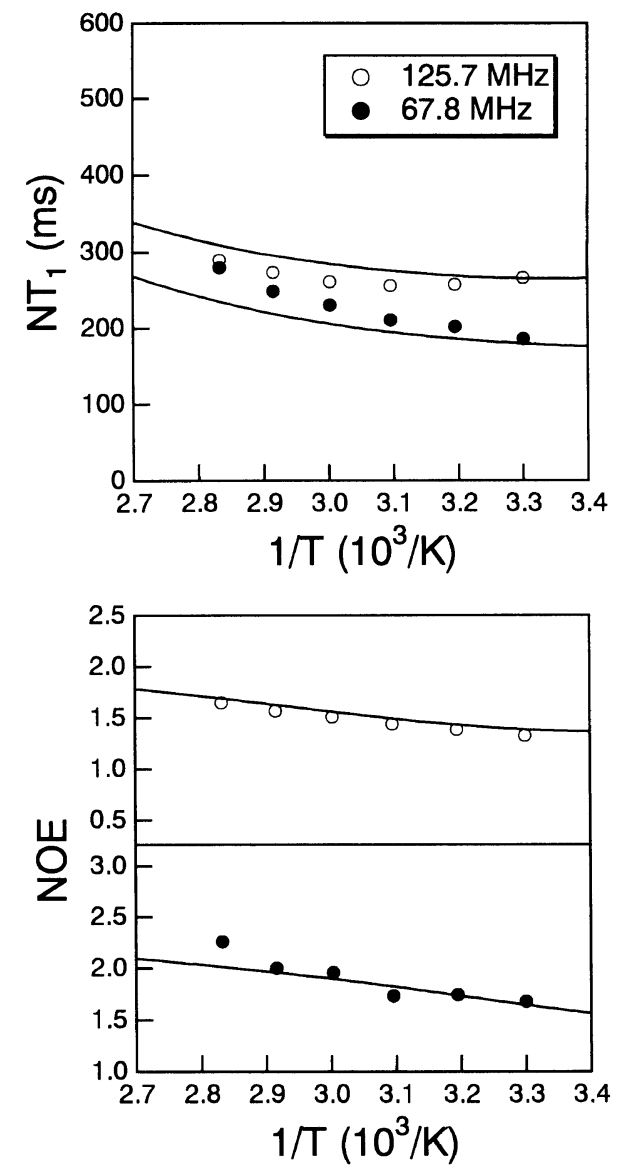

Figure 9. Temperature dependences of $T_{1}$ and NOE for $\mathrm{C}_{4}$ carbon in pyridyl ring of iso-P2VP. Solid lines denote the theoretical values of the restricted rotational model with the parameters listed in Table IV.

Table IV. Fitting parameters of the restricted rotational model for pyridyl ring carbon $\left(\mathrm{C}_{4}\right)$ of iso- $\mathrm{P} 2 \mathrm{VPCl}$ and iso- $\mathrm{P} 2 \mathrm{VP}$

\begin{tabular}{lcccc}
\hline & $E_{\mathrm{ir} /} / \mathrm{kJ} \mathrm{mol}^{-1}$ & $10^{13} A_{\mathrm{ir}} / \mathrm{s}$ & $C$ & $\alpha / \mathrm{deg}$ \\
\hline P2VPCl & 21.0 & 0.18 & 1.6 & $28-30$ \\
P2VP & 20.0 & 0.15 & 2.1 & $37-39$ \\
\hline
\end{tabular}

\section{REFERENCES}

1. H. Dautzenberg, W. Jaeger, J. Kotz, B. Philipp, Ch. Seidel, and D. Stscherbina, "Polyelectrolytes-Formation, Characterization and Application," Hanser Publishers, Munich, 1994.

2. M. Hara, Ed., "Polyelectrolytes," Marcel Dekker, New York, N.Y., 1993.

3. T. Odijk, J. Polym. Sci., Polym. Phys. Ed., 15, 477 (1977); J. Skolnick and M. Fixman, Macromolecules, 10, 944 (1977).

4. M. Le Bret, J. Chem. Phys., 76, 6243 (1982); M. Fixman, ibid., 76, 6346 (1982).

5. F. A. Bovey and P. A. Mirau, "NMR of Polymers," Academic Press, San Diego, 1996.

6. W. H. Stockmayer, Pure Appl. Chem., 15, 539 (1967).

7. J. P. Runt and J. J. Fitzgerald, Ed., "Dielectric Spectroscopy of Polymeric Materials: Fundamentals and Applications," The American Chemical Society, Washington, D.C., 1997.

8. M. A. Winnik, Ed., "Photophysical and Photochemical Tools in Polymer Science: Conformation, Dynamics, Morphology," D. Reidel Pub., Dordrecht, 1985.

9. C. Chachaty, A. Forchioni, and J. Ronfard-Haret, Makromol. Chem., 173, 213 (1973).

10. D. Ghesquière, B. Ban, and C. Chachaty, Macromolecules, 10, 743 (1977).

11. D. Ghesquière and C. Chachaty, Macromolecules, 11, 246 (1978).

12. S. Ravindranathan and D. N. Sathyanarayana, Macromolecules, 28, 2396 (1995).

13. R. Dejean de la batie, F. Lauprêtre, and L. Monnerie, Macromolecules, 21, 2045 (1988).

14. C. K. Hall and E. Helfand, J. Chem. Phys., 77, 3275 (1982).

15. T. A. Weber and E. Helfand, J. Phys. Chem., 87, 2881 (1983).

16. S. Yamazaki, E. Okada, Y. Muroga, I. Noda, and A. Tsutsumi, Polym. J., in press.

17. G. Natta, G. Mazzanti, P. Longi, G. Dall'asta, and F. Bernardini, J. Polym. Sci., 51, 487 (1961).

18. K. Matsuzaki and T. Sugimoto, J. Polym. Sci. A-2, 5, 1320 (1967).

19. S. Arichi, Bull. Chem. Soc. Japan., 39, 439 (1966).

20. D. Doddrell, V. Glusko and A. Allerhand, J. Chem. Phys., 56, 3683 (1972).

21. J. A. Pople and M. S. Gordan, J. Am. Chem. Soc., 89, 4253 (1967).

22. F. Mata, M. J. Quintana, and G. O. Sorensen, J. Mol. Struct., 42, 1 (1977).

23. J. R. Lyerla and G. C. Levy, Top. Carbon-13 NMR Spectrosc., 1, 79 (1974).

24. P. Parhami and B. M. Fung, J. Am. Chem. Soc., 107, 7304(1985)

25. O. W. Howarth, J. Chem. Soc., Faraday Trans. 2, 75, 863 (1979).

26. W. Gronski and N. Murayama, Macromol. Chem., 179, 1521 (1978).

27. E. Helfand, J. Chem. Phys., 54, 4651 (1971).

28. H. A. Kramers, Physica, 7, 284 (1940).

29. D. J. Gisser, S. Glowinkowski, and M. D. Ediger, Macromolecules, 24, 4270 (1991).

30. D. R. Lide, Ed., "CRC Handbook of Chemistry and Physics," CRC Press, Inc., Boca Raton, FL, 1995.

31. A. Spyros, P. Dais, and F. Heatley, Macromolecules, 27, 6207 (1994).

32. A. Guillermo, R. Dupeyre, and J. P. Cohen-Addad, Macromolecules, 23, 1291 (1990). 